\title{
Personalien und Tagesnachrichten
}

Professor Alfred Marchionini (Freiburg) hat einen Ruf als Leiter der Hautklinik am.

Musterkrankenhaus nach Ankara (Türkei) erhalten, dem er 1938 folgen wird.

Professor Paul Mulzer (Hamburg) wurde zum Lupusbeauftragten f ür Groß-Hamburg ernannt.

Geheimrat Bernhard Nocht (früher Hamburg, jetzt Wiesbaden) beging seinen 80. Geburtstag;

ihm wurde der Adlerschild des Deutschen Reiches ver-liehen.

Professor Oustav Giemsa feierte den 70. Geburtstag; er wurde von der Deutschen

Tropenmedizinischen Gesellschaft zum Ehrenmitglied gewählt.

Professor Guido Miescher (Zurich) wurde von der Deutschen Gesellschaft fur Lichtforschung zum Ehrenmitglied gewählt.

Professor Dörffel (Halle) wurde von der Ungarischen Dermatologischen Gesellschaft zum korrespondierenden Mitglied gewählt.

Professor Nanta (Toulouse) wurde zum Direktor der dortigen Hautklinik ernannt.

Dr. J. Merenlender (Warschau) wurde von der Ungarischen Dermatologischen Gesellschaft zum korrespondierenden Mitglied gewählt.

Arthur DougL·s Heath (London), seit 1926 Vorsitzender der Britischen Dermatologischen Gesellschaft und seit mehreren Jahren auch stellvertretender Vorsitzender der dermatologischen Abteilung der Royal Society of Medicine, ist am 8. Mai verschieden.

Dr. George R. Simmons (Chicago), Mitbegründer der Archives of Dermatology and Syphilology, und Dr. S. Pollitzer, einer der bekanntesten Derma-tologen in Neuyork, sind gestorben.

Der X. Internationale Oermatologenkongreß (ursprünglich fur Madrid vorgesehen) ist endgültig auf Sommer 1940 nach Neuyork verlegt worden; Vorsitz Professor Oliver S. Ormsby (Chicago), Generalsekretär Dr. Paul O’Leary (Rochester, Minn.).

Am 10. Juni wurde in Neuyork unter dem Vorsitz von Dr. George M. Mac Kee die Society of Investigative Dermatology gegründet, die eine neue Zeit-schrift, das Journal of Investigative Dermatology, herausgeben wird; Schrift-leitung: 962 Park Avenue. In den englischen Kolonien werden 2 Millionen Lepröse geschätzt. 